\title{
Action planning with two-handed tools
}

\author{
Arvid Herwig $\cdot$ Cristina Massen
}

Received: 29 April 2008 / Accepted: 1 October 2008 / Published online: 6 November 2008

(C) The Author(s) 2008. This article is published with open access at Springerlink.com

\begin{abstract}
In tool use, the intended external goals have to be transformed into bodily movements by taking into account the target-to-movement mapping implemented by the tool. In bimanual tool use, this mapping may depend on the part of the tool that is operated and the effector used (e.g. the left and right hand at the handle bar moving in opposite directions in order to generate the same bicycle movement). In our study, we investigated whether participants represent the behaviour of the tool or only the effector-specific mapping when using two-handed tools. In three experiments, participants touched target locations with a two-jointed lever, using either the left or the right hand. In one condition, the joint of the lever was constant and switching between hands was associated with switching the target-to-movement-mapping, whereas in another condition, switching between hands was associated with switching the joint, but the target-to-movement-mapping remained constant. Results indicate pronounced costs of switching hands in the condition with constant joint, whereas they were smaller with constant target-to-movement mapping. These results suggest that participants have tool-independent representations of the effector-specific mappings.
\end{abstract}

\section{Introduction}

Much of our everyday behaviour consists of goal directed movements. Some of these movements are directly exe-

\footnotetext{
A. Herwig $(\bowtie) \cdot$ C. Massen Department of Psychology, Max-Planck-Institute for Human Cognitive and Brain Sciences, Stephanstraße 1a, 04103 Leipzig, Germany

e-mail: herwig@cbs.mpg.de
}

cuted to a desired target in external space, for example moving the hand and fingers to a cup of tea. However, there are many goals which cannot be attained directly (e.g. open a can with your hands only). Therefore, humans and some animals have established a culture in which they extend their physical abilities by using tools. Whereas animals typically use quite simple tools that serve as mere extensions of the effectors, tools invented and manufactured by humans also include highly complex technical devices and machines that afford quite sophisticated knowledge on the side of the user.

A multitude of aspects has to be taken into account in action planning with tools. For instance, using a can opener (also known as tin opener) requires semantic knowledge concerning the relationship between the tool and its function. If this knowledge is missing, a can opener cannot be linked to the appropriate object (i.e. a can) to act upon. Moreover, in tool use the desired effect in external space (e.g. opening a can) typically has to be transformed into corresponding bodily movements (e.g. movement of the hand at the handle of a can opener) - that is procedural knowledge has to be acquired and represented, too. To acquire this procedural knowledge, the target-to-movement transformation of a specific tool has to be learned. This transformation is a function that assigns each external target that is reachable with the tool, a specific bodily movement. If this bodily movement is executed, it causes the tool to hit or affect the target in the desired way. In the following, we will, in analogy to the well-known term of a stimulus-response mapping, refer to this function as the target-tomovement mapping defined by a tool.

The question as to how goal directed actions are controlled is a key question in cognitive psychology. However, exploring the mental processes guiding actions that are performed with tools has attracted interest only recently. The 
majority of these recent studies examine a special case of tool-use actions, namely tool-use actions that are performed with one hand like handling levers, grippers, or tweezers (e.g. Kunde, Müsseler, \& Heuer, 2007; Beisert, Massen, \& Prinz, 2008; Massen \& Prinz, 2007a, b; Müsseler, Kunde, Gausepohl, \& Heuer, 2008). Yet, many everyday actions with tools are performed with two hands, either simultaneously, like cutting grass with a scythe, or in succession, like cutting a twig with a wire saw. In the following, we will use the term bimanual or two-handed tool use for actions that are performed with either the left or right hand, not simultaneously.

In this study, we focus on the mental processes and representations underlying the ability to use two-handed tools. More specifically, we asked whether people build up mental models representing the behaviour of the physical tool at hand, or whether tool-use actions are guided by more frugal, tool-independent representations of the required effector-specific mapping of bodily movements into associated tool effects (i.e. the target-to-movement mapping).

\section{Action planning with tools}

In each tool-use action, two main components have to be taken into account, before the bodily movement can be computed: The intended goal state or desired effect in external space (e.g. hitting a nail on the head) and the specific target-to-movement mapping which is realized by the tool (e.g. the transformation of hand movements at a hammer's handle into the desired movement of the hammer's head). A recent study by Massen and Prinz, (2007a) investigated the process of action planning with tools. Participants had to touch target locations with a horizontal lever that was movable around one of two possible pivotal points. In each trial, one of the two pivotal points and one of the two targets were indicated by lights, and participants had to move the lever in the correct direction to hit the target location, taking into account the active pivotal point. Using a precuing method (cf. Rosenbaum, 1980, 1983), the authors were able to show a large benefit for precuing the target-tomovement mapping (i.e. the active pivotal point), whereas precuing the target location was much less effective. A further study (Massen \& Prinz, 2007b) extended this result by showing that participants performed faster and more accurately on a given trial $n$, if the target-to-movement mapping of trial $n-1$ was repeated (but the target changed), than on trials where the target was repeated (but the target-to-movement mapping changed). Taken together, the results reported so far indicate that humans have an internal model of the tool-specific mapping between external effect and associated bodily movement, which is accessed early in the process of action planning before further parameters (e.g. the distal target location) are specified. Yet, the results give rise to the question whether such an internal model is specific to the tool at hand, or whether it is more abstract in the sense that it can be used by different tools sharing a common physical mechanism.

Especially relevant in this context is a study by Beisert et al. (2008) investigating the question if the cognitive representation of such a target-to-movement mapping is bound to a specific tool. Using a tool-switching paradigm, in which participants switched between tools that implement either a compatible movement-effect mapping (pincers and tweezers) or an incompatible one (clothespin and clip), the authors were able to show that there is a benefit for mapping repetitions in comparison to mapping switches even when the tool changes. Unfortunately, results about toolrepetitions were not reported, because the study mainly focused on tool-switching with or without switching the target-to-movement mapping. However, a repetition of the tool, which came along with a repetition of the mapping, yielded the shortest reaction times (M. Beisert, personal communication). Altogether, these results provide two bits of information. The first is that the cognitive representation of a target-to-movement mapping is relatively abstract, the same internal model being suitable for different tools as long as they share a common target-to-movement transformation (e.g. pincers and tweezers). However, as a second bit of information, the benefit of repeating the same tool can be taken as a first tentative hint that people may also acquire representations that are specific to the tool at hand.

Yet, to directly test if people use tool-specific mental models, one would have to independently manipulate tool as well as mapping repetitions and switches. At first sight, this seems to be a hopeless endeavour, since it is hard to imagine how to use one and the same tool with different target-to-movement mappings. However, this problem can be solved by taking into account that there are tools which can be operated with two hands. Imagine for example a horizontal lever with two handles left and right, that can be moved up or down with the left or right hand, respectively (see Fig. 1). If the pivot of the lever is close to its left handle, the left handle has to be moved down to touch a target that is located above the lever (incompatible target-tomovement mapping). However, the same lever requires an upward movement to touch the target above the lever if the right handle is used (compatible target-to-movement mapping).

Action planning with two-handed tools

Despite the fact that there is a considerable amount of work on bimanual action planning without tools (e.g. Heuer, 1993; Heuer \& Klein, 2006; Diedrichsen, Ivry, Hazeltine, Kennerly \& Cohen, 2003; Spijkers, Heuer, Kleinsorge, \& van der Loo, 1997; Kunde \& Weigelt, 2005), only little is 


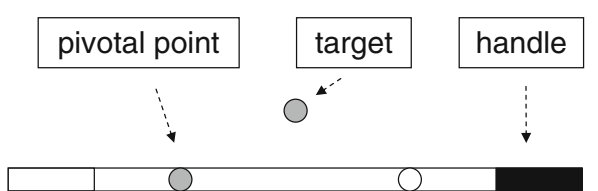

$\bigcirc$

a) instruction display

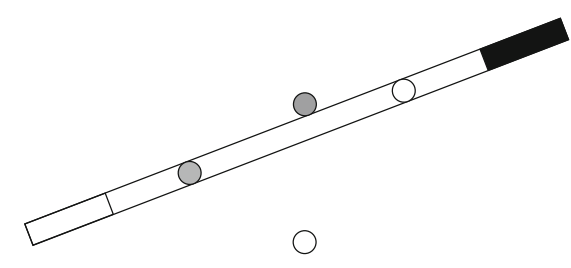

b) effect display

Fig. 1 Sequence of display events in the lever paradigm

known about how people plan and represent tool-use actions performed with both hands. Imagine for example riding a bicycle while holding the handle bar with either the left or the right hand. If you want to ride left and you are using your right hand, you have to push the handle bar away from the body (i.e. arm extension). However, using your left hand requires pulling the handle bar towards the body (i.e. arm flexion) to turn leftwards. The same holds also true for responses with a steering wheel, which have been studied in several experiments exploring the Simon effect or stimulus-response compatibility (e.g. Guiard, 1983; Proctor, Wang, \& Pick, 2004; Wang, Proctor, \& Pick, 2003, 2007). These examples show that the target-tomovement mapping may depend on the part of the tool that is operated and the effector used.

Up to now, it is an open question how such two-handed tool-use actions are represented. From a theoretical point of view, two possibilities of representing a tool handled with two hands can be distinguished. The first possibility is that people develop a higher-order representation of the behaviour of a tool, with representations of the associated effector-specific mappings at a subordinate level. The advantage of such a tool-based representation that incorporates all possible behaviours of the tool might be that it enables a certain degree of dynamic invariance. Whenever an action with this specific tool has to be executed, the very same internal model can be accessed whatever the current situational constraints (e.g. the effector used) are. In this view, the corresponding effector-specific mapping would be dynamically tuned in at a later stage in movement planning. Such a representation might further help to extend the tool's range of application, i.e. knowing that a given tool behaves in different ways depending for example on the part that is handled might allow its usage to solve new problems. In his review on the speciality of human tool use
Johnson-Frey (2003) argues, that it is exactly this ability to represent (and reason about) objects' physical properties and their causal interactions with the world, that distinguishes human tool use from the one exhibited by other species.

On other hand, it might also be the case that people do not represent the physical tool and its behaviour, but simply the target-to-movement mapping that is needed in the current situation. Such a mapping-based representation would have the advantage to be frugal, since the same representation can be used for different tools (and effectors) as long as they share a common target-to-movement mapping.

Overview of the present study

To investigate how two-handed tool-use actions are represented, we extended the experimental tool-switching paradigm developed by Massen and Prinz (2007a). In the twohanded tool-use task, participants have to touch target locations with a two-jointed lever displayed on the computer screen. The lever can be moved with either the left or the right hand - that is, the left hand controls the left handle of the lever, the right hand the right handle. In each trial a pivotal point, a target location, and a handle of the lever is specified, and participants have to indicate in which direction to move the required handle to touch the target (see Fig. 1). Importantly, in some conditions of trial transitions, a hand switch is associated with switching the target-tomovement mapping but repeating the tool mechanics (i.e. repeating the active pivotal point). For instance, if the left pivotal point is active and a response with the right hand is requested, the right handle has to be moved upwards to touch the upper target point (compatible target-to-movement mapping). In contrast, if a response with the left hand is requested, the left handle has to be moved downwards to hit the upper target point (incompatible target-to-movement mapping). However, in other conditions of trial transitions, a hand switch involves switching the tool mechanics (i.e. switching the active pivotal point) but repeating the current target-to-movement mapping. For instance, if a response with the right hand is requested and the left pivotal point is active, the right handle has to be moved upwards to touch the upper target point. Likewise, if a response with the left hand is requested and the right pivotal point is active, the left handle has to be moved upwards to touch the upper target point.

The abovementioned possibilities of representing a tool handled with two hands allow different predictions with respect to the outcome of this manipulation. First of all, consider that each pivot of the lever apparatus defines a specific tool (either one with a compatible or one with an incompatible target-to-movement mapping). If one assumes that people build up a tool-based representation, switching 
the hand while repeating the tool (i.e. the pivot) should cause less hand switch costs than a hand switch associated with a tool switch. This is because in the former case, the same higher order representation of the tool can be used for the left as well as the right hand. Yet, if people develop and use a mapping-based representation, one would expect hand switch costs to be reduced, when a hand switch comes along with repeating the target-to-movement mapping, since in this case the same representation of the target-tomovement mapping can be used for both hands. ${ }^{1}$

Three experiments were conducted to test these predictions. In Experiment 1, participants had to indicate in which direction to move the lever's handle by pressing one of two response buttons on either a left or a right placed response device in front of them. In Experiment 2, we used a more natural setting, in which participants had to move a left or right joystick in the appropriate direction to control the lever displayed on the computer screen. Finally, in Experiment 3 participants were required to move the left or right handle of a real lever to touch one of two target locations. Moreover, in Experiments 2 and 3 the critical manipulation was made between-subjects instead of within-subjects like in Experiment 1. Therefore, the pivotal point was always constant in one group of subjects (i.e. tool-repetition and mapping-switch) whereas it changed with a hand switch in another group of subjects (i.e. tool-switch and mappingrepetition).

\section{Experiment 1}

In Experiment 1, a horizontal lever, that was movable around one of two possible pivotal points with either the left or right handle, was displayed on the computer screen. In each trial, one of two target locations, the active pivotal point, and the valid handle of the lever were specified. Participants' task was to touch the indicated target location with the lever by moving the appropriate handle of the lever in the appropriate direction (up or down), taking into account the active pivotal point. The displayed lever was movable by pressing the upper or lower key on one of two response devices that were placed on the left (to control the left handle) and on the right hand side (to control the right handle) in front of the participant.

We compared four different conditions of trial transitions from trial $n-1$ to trial $n$ (see Fig. 2), in which either (a) only the handle was repeated, mapping and pivotal point changed; (b) only the active pivotal point was repeated,

\footnotetext{
${ }^{1}$ Note that the influence of repeating and switching the movement direction (toward or away from the body) is not the focus here and that the relevant transition conditions comprise movement repetitions and switches in equal measure.
}

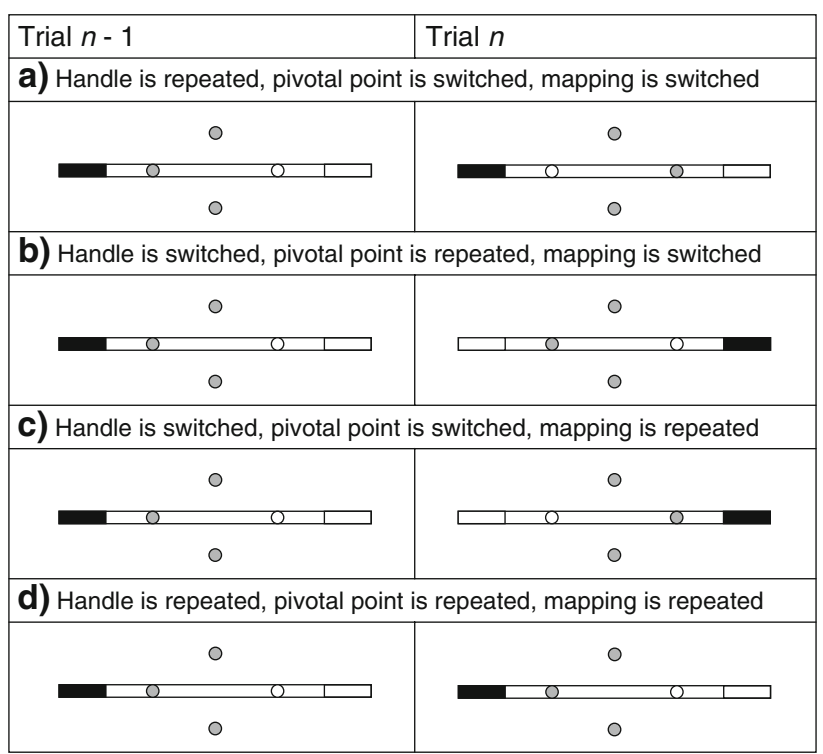

Fig. 2 Illustration of the transition conditions in Experiment 1. In each trial, one of two target locations, the active pivotal point, and the valid handle of the lever are specified. To simplify matters, both target points are displayed in the illustration

handle and mapping changed; (c) only the target-to-movement mapping was repeated, handle and pivotal point changed; (d) all three components were repeated. With regard to the addressed question, the essential comparison was between transition condition (b) and (c), where either the tool (i.e. the pivotal point) or the target-to-movement mapping was repeated while the handle was switched.

Method

\section{Participants}

A total of 12 right-handed participants ( 7 female, 5 male; mean age $=24.1$ years), with normal or corrected-to-normal vision from the subject pool of the Max-Planck-Institute in Leipzig, participated in Experiment 1. Participants were naive with respect to the purpose of the experiment and were paid 7 Euro for their participation.

\section{Apparatus and stimulus display}

The display and timing were controlled by an IBM compatible computer, interfaced to a 17 inch VGA display, which was connected to two separated response pads via the parallel port. The two response devices were placed on the left and right hand side in front of the participants in a distance of $55 \mathrm{~cm}$ from each other. On each response pad, three touch-sensitive plates $(2.5 \mathrm{~cm}$ in width $\times 1.8 \mathrm{~cm}$ in length $)$ were arranged from top to bottom in the horizontal plane, in a distance of $0.5 \mathrm{~cm}$ from each other. The middle plates 
served as the home keys. The participants performed the responses with their left and right index fingers, which were to be laid down on the home keys before each trial started. The lever display was approximately $13 \mathrm{~cm} \times 4 \mathrm{~cm}$ in overall size, drawn in black on a white background.

The lever display consisted of one lever with two handles, two pivotal points, and two target points (see Fig. 1). Targets and pivotal points were drawn as unfilled circles $(0.3 \mathrm{~cm}$ in diameter). Target points were placed $2 \mathrm{~cm}$ above and below the lever, orthogonal to the lever's midpoint. The two pivotal points were drawn on the left and right part of the lever with a distance of $3 \mathrm{~cm}$ to the lever's midpoint. Due to the fact that target points were situated in equal distance from both pivotal points, the amplitude of the imagined movement of the lever's handle was larger for the compatible mapping (e.g. left pivotal point and right handle) than for the incompatible mapping (e.g. left pivotal point and left handle). The two handles of the lever were drawn on the right and left side. Their inner boundaries were at a distance of approximately $5.5 \mathrm{~cm}$ to the lever's midpoint.

\section{Design and procedure}

Participants were first informed about the task, which was to touch the indicated target location with the lever by moving the appropriate handle of the lever in the appropriate direction (up or down), taking into account the active pivotal point. They were further instructed to place the left and right index finger on the appropriate home key, which triggered the beginning of the trial. After a preparation interval of $1,000 \mathrm{~ms}$, the instruction display was presented on the screen. On each trial, one target and one pivotal point were drawn in red colour, and one handle was drawn in black colour to inform the participant about the target point to touch, the active pivotal point, and the valid handle. Participants were required to respond with the index finger of the indicated hand while the other index finger had to stay on the home key. Reaction time was defined as the time from the onset of the lever display to the first release of the home key. Movement time was measured from the first release of the home key to the first touch of one of the other keys (above or below the home key). Touching the upper or lower key triggered the presentation of an effect display for $500 \mathrm{~ms}$, which showed the lever, moved around the active pivotal point, so that the participant was able to watch whether the instructed target point was touched by the lever. If the participant responded with the wrong hand or if the instructed target point was not touched, an error feedback was presented and the participant was asked to press the correct button. Then the next trial started.

All in all, there were eight different combinations of pivotal points (left/right), target points (up/down), and handles (left/ right). Hence, 64 types of transitions between trials are possi- ble, which can be reduced to four relevant transition conditions (see Fig. 2). Each of the eight trial types and each of the 64 possible transitions between trials appeared equally often and in a randomized order for each individual participant.

The experiment consisted of a practice block of 20 trials, after which subjects went through four experimental blocks of 128 trials (plus one additional, later discarded starting trial to make sure that all transition conditions appeared equally often). After each block, participants had the opportunity to take a short break. The whole experiment took approximately $35 \mathrm{~min}$.

\section{Results}

Dependent variables were reaction times for correct responses and error rates. We also calculated movement times but as there were no effects of interest for this variable in all three experiments, we do not report these results in the following. Violations of sphericity were corrected using the Huynh-Feldt $\varepsilon$ (to facilitate reading, the uncorrected degrees of freedom are provided). Throughout the article, we adopt a significance level of 0.05 .

Individual median reaction times were calculated for each of the four transition conditions and are depicted together with the mean error rates in Fig. 3.

\section{$R T s$}

A repeated-measures analysis of variance on reaction times with the factor transition condition indicated a significant main effect of transition condition $(F(3,33)=16.0$; MSE $=11,746 ; \varepsilon=.48 ; p<0.001)$. Essentially, as revealed by a single comparison, the $50 \mathrm{~ms}$ difference between

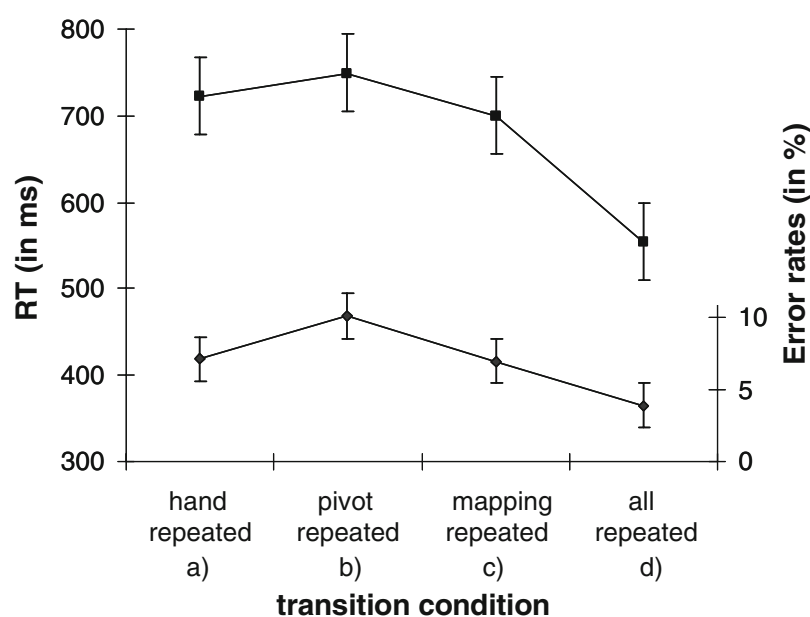

Fig. 3 Mean RT (in ms) and mean error rates (in \%) as a function of transition condition in Experiment 1. Within-subject error bars show 95\% confidence intervals and were computed according to Loftus and Masson (1994) 
transition conditions (b) and (c) reached statistical significance $(t(11)=2.6 ; p<0.05$; two-tailed), indicating that participants were significantly faster with a mapping-repetition than a pivot-repetition while switching hands.

\section{Error rates}

The analysis of error rates yielded a significant main effect of transition condition $(F(3,33)=10.5 ; \quad \mathrm{MSE}=7.2$; $p<0.001)$. The difference between transition conditions (b) and (c) reached again statistical significance $(t(11)=2.4$; $p<0.05$; two-tailed), indicating that participants made significantly less response errors with a mapping-repetition than a pivot-repetition while switching hands.

\section{Discussion}

In Experiment 1, we were interested in the question how two-handed tool-use actions are represented. More specifically, we wanted to know whether such actions are represented with respect to the underlying behaviour of the tool or whether mainly the target-to-movement mapping is considered in action planning. The results of the reaction time and error analysis are clear-cut. They show that a hand switch is easier when the target-to-movement mapping is repeated while the pivotal point is switched, than when the pivotal point is repeated while the target-to-movement mapping is switched. This observation suggests that participants use a mapping-based representation that is quite independent from the current functioning of the tool.

However, the results we obtained might be influenced by two aspects of the experimental situation that could have worked against the formation of a tool-based representation. First, controlling a displayed lever by pressing buttons might be regarded as a somewhat artificial tool-use task. Accordingly, the benefit of repeating a target-to-movement mapping might be limited to situations, where participants have to simulate tool use instead of performing real tooluse actions. Thus, in Experiments 2 and 3 we transferred our lever paradigm into a more naturalistic setting by changing the response apparatus. Second, if one assumes that the formation of a tool-based representation might take some time and experience with one and the same tool, manipulating tool and mapping switches within-subjects might interfere with this formation process. Therefore, in Experiments 2 and 3 we decided to manipulate tool and mapping switches between-subjects.

\section{Experiment 2}

In Experiment 2, participants had to move a left or right joystick of a response device in the appropriate direction to control the lever displayed on the computer screen (see Fig. 4).

Again, the task was to touch one of two target points with the lever by moving the correct handle in the appropriate direction, taking into account the active pivotal point. Importantly, in contrast to Experiment 1, the pivotal point and hence the tool mechanics was constant for one group of participants (see Fig. 5, upper part), whereas in the other group, the target-to-movement mapping was constant. In this group, switching hands was always associated with switching the pivotal point (see Fig. 5, lower part).

In addition, a further factor was included to control for amplitude differences of the imagined movement of the lever's handle. As pointed out earlier, placing the target points in equal distance from both pivotal points leads to

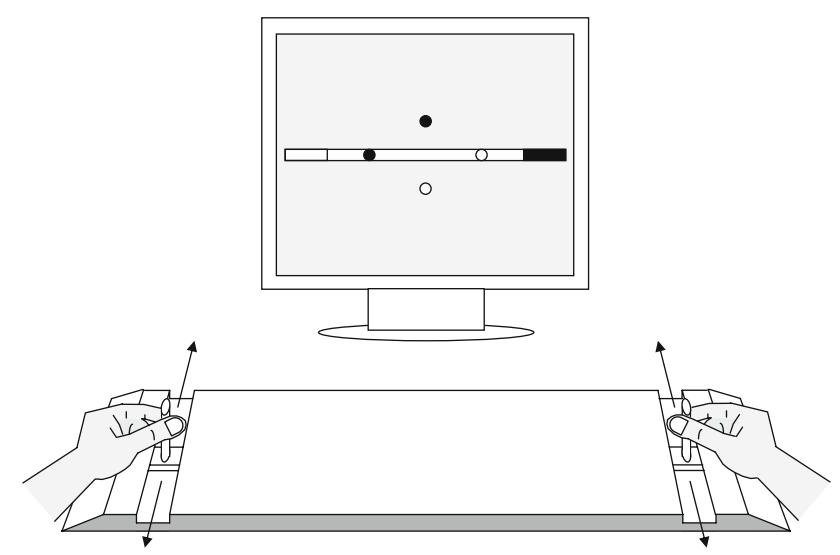

Fig. 4 Response device used in Experiment 2

\begin{tabular}{|c|c|c|}
\hline Trial $n-1$ & & Trial $n$ \\
\hline \multicolumn{3}{|c|}{ 1) Pivotal point same / Mapping switched } \\
\hline 1a) & 0 & 0 \\
\hline & ० & ○ \\
\hline 1b) & o & 0 \\
\hline & o & ○ \\
\hline \multicolumn{3}{|c|}{ 2) Pivotal point switched / Mapping same } \\
\hline 2a) & 0 & 0 \\
\hline 2 & $\begin{array}{lll} & 0 & \\
0 & & \end{array}$ & $\longrightarrow 0$ \\
\hline 2b) & ○ & O \\
\hline$m$ & $\begin{array}{llll} & 0 & \\
0 & \end{array}$ & 0 \\
\hline
\end{tabular}

Fig. 5 Illustration of the transition groups in Experiments 2 and 3. In each trial, one of two target locations, the active pivotal point, and the valid handle of the lever are specified. To simplify matters, both target points are displayed in the illustration 
larger movement amplitudes for the lever handle in the conditions with a compatible target-to-movement mapping as compared to those with an incompatible one. As a consequence, participants might have estimated the time it takes to reach the target position differently for the compatible and incompatible mapping. This might be problematic, since Kunde (2003) showed that response latencies increase with increasing the duration of an associated effect, suggesting that it takes longer to anticipate a long-lasting than a short-lasting effect. Thus, we used two different lever displays which were varied between-subjects-one with the target points placed in equal distance to the pivotal points and another one with equal amplitudes of the imagined movement of the lever's handle.

\section{Method}

\section{Participants}

A total of 48 right-handed participants ( 31 female, 17 male; mean age $=23.6$ years), with normal or corrected-to-normal vision from the subject pool of the Max-Planck-Institute in Leipzig, participated in Experiment 2. They were randomly assigned to two groups of 24 participants each. Participants were naive to the purpose of the experiment and were paid for their participation.

\section{Apparatus and stimulus display}

The new response device consisted of two vertical movable sticks, which were mounted in a distance of $55 \mathrm{~cm}$ on a plastic plate (see Fig. 4). Each stick rested in the middle position, which served as the home position. The participants performed their responses with their left and right index finger and thumb gripping the upper end of the left and right stick. Both sticks could be moved independently $3.5 \mathrm{~cm}$ away or towards the participant. The response device registered the stick leaving the home position, arriving at the end position, and returning back to the home position. The lever display was approximately $23 \mathrm{~cm} \times 7 \mathrm{~cm}$ in overall size.

Two different lever displays were used. One was arranged like the lever display in Experiment 1 that is the target points were situated in equal distance from both pivotal points. On the other lever display, target points were placed closer to the valid handle of the lever, thus ensuring that the amplitude of the imagined movement of the lever's handle would be the same for compatible and incompatible target-to-movement mappings.

\section{Design and procedure}

Participants were first informed about the task. They were further instructed to grip the left and right stick between the index finger and thumb of their left and right hand. After a preparation interval of $500 \mathrm{~ms}$, the instruction display was presented on the screen. On each trial one target and one handle were specified to inform the participant about the task. Participants were required to respond by moving the stick with the indicated hand while the other stick had to stay on the home position. Reaction time was defined as the time from the onset of the lever display to the leaving of the home position (i.e. a movement of the stick about $1 \mathrm{~mm}$ off the neutral position). Movement time was measured from leaving the home position to the arrival at the end position, which triggered the $500 \mathrm{~ms}$ presentation of an associated effect display. The effect display showed the lever, moved around the active pivotal point, so that the participant was able to watch whether the instructed target point was touched by the lever. If the participant responded with the wrong hand or if the instructed target point was not touched, an error feedback was presented, and the participant was asked to move the correct stick to the correct end position. The next trial started after returning back to the home position.

There were two different groups of participants (see Fig. 5). In the first group, a hand switch was always associated with repeating the pivotal point but switching the target-to-movement mapping-that is the tool mechanics did not change over the whole experiment. This pivot-repetition group was further subdivided into two subgroups. In the first subgroup, the active pivotal point was always drawn on the left side of the lever, whereas in the second subgroup, it was always on the right side of the lever (see Fig. 5, Subgroups 1a and 1b, respectively). In the second group, switching between hands was associated with switching the pivotal point, such that the target-to-movement mapping remained constant throughout the whole experiment. Again, this mapping-repetition group was subdivided into two subgroups. In the first subgroup, the active pivotal point was always on the opposite side to the valid handle of the lever (compatible mapping) whereas in the second subgroup it was always on the side of the valid handle (incompatible mapping) (see Fig. 5, Subgroups 2a and $2 b$, respectively). For each group, there were four different combinations of target points (up/down) and handles (left/right). Hence, there are 16 possible transitions between trials, which can be reduced to two relevant types of transitions between trials (hand-repetition/handswitch). Each of the four trial types and each of the 16 possible trial transitions appeared equally often and in a randomized order for each individual participant. Half of the participants saw the lever display with the target points placed in equal distance to the pivotal points and the other half of participants received the lever display with equal amplitudes of the imagined movement of the lever's handle. 
Fig. 6 Mean RT (in ms) on the left panel and mean error rates (in \%) on the right panel as a function of transition condition and transition group in Experiment 2. Error bars show standard errors

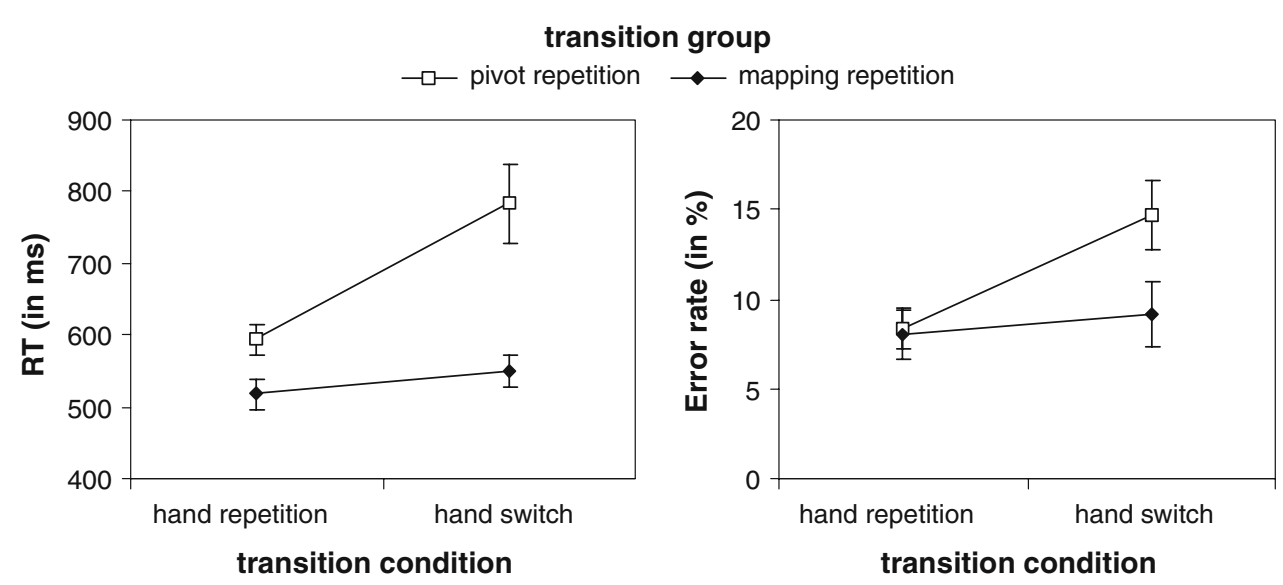

The experiment consisted of a practice block of 16 trials, after which subjects went through four experimental blocks of 64 trials (plus one additional, later discarded starting trial). After each block, participants had the opportunity to take a short break. The whole experiment took approximately $30 \mathrm{~min}$.

\section{Results}

Trials in which participants responded in the preparation interval or responded with both hands (1.2\%) were excluded from analysis. All reactions initiated with the wrong hand and/or in the wrong movement direction, irrespective of whether they were corrected or not, were defined as errors. Individual median reaction times for correct responses and error rates were then calculated for hand-repetitions and hand-switches for each of the two transition groups (pivot-repetition group vs. mapping-repetition group). They are depicted in Fig. 6.

\section{$R T s$}

An ANOVA was run on the reaction times with the withinsubject factor transition condition (hand-switch/hand-repetition) and the two between-subject factors transition group (pivot-repetition/mapping-repetition) and display group (different-amplitude display/same-amplitude display). The analysis yielded a significant main effect of transition condition $(F(1,44)=30.2$; MSE $=9,745 ; p<0.001)$, that was due to shorter reaction times on trials with a hand repetition, and a significant interaction of transition condition $\times$ transition group $(F(1,44)=15.3$; $\mathrm{MSE}=9,745$; $p<0.001)$. Figure 6 reveals the RT difference between hand repetition trials and hand switch trials to be greater in the pivot-repetition group (190 ms) than in the mappingrepetition group $(32 \mathrm{~ms})$. There was also a main effect of transition group $(F(1,44)=12.8 ; \mathrm{MSE}=45,114 ; p<0.01)$, that was due to shorter reaction times in the mapping-repe- tition group. Neither the main effect of the factor display group nor any interaction with this factor reached statistical significance (all $F \mathrm{~s}<1$ ).

As a follow up analysis, two separate ANOVAs were run for the pivot-repetition group as well as for the mapping-repetition group including the within-subject factor transition condition and the between-subject factor transition subgroup. The ANOVA for the pivot-repetition group yielded a significant main effect of transition condition $(F(1,22)=23.1 ;$ MSE $=18,697 ; p<0.001)$, demonstrating shorter reaction times on trials with a hand repetition. Neither the main effect of transition subgroup (left pivotal point/right pivotal point) nor the interactions with this factor reached statistical significance (all $F_{\mathrm{s}}<1$ ). The ANOVA for the mapping-repetition group showed a significant main effect of transition condition $(F(1,22)=16.8$; MSE $=721 ; p<0.001)$ and an almost significant main effect of transition subgroup (compatible mapping/incompatible mapping) $(F(1,22)=4.0 ; \mathrm{MSE}=19,588 ; p=0.06)$, that was due to shorter reaction times for the subgroup with the compatible mapping (493 vs. $574 \mathrm{~ms}$ ). However, the interaction between transition condition and transition subgroup was not significant $(F(1,22)=1.3$; MSE $=721)$.

\section{Error rates}

The analysis on error rates with the within-subject factor transition condition and the two between-subject factors transition group and display group yielded a significant main effect of transition condition $(F(1,44)=13.4$; MSE $=24.9 ; p<0.01)$, which indicated less response errors on trials with a hand repetition than on trials with a hand switch. The effect of transition condition was qualified by a significant interaction of transition condition $\times$ transition group $(F(1,44)=6.5 ; \mathrm{MSE}=24.9 ; p<0.05)$. Moreover, the factor display group reached statistical significance $(F(1,44)=6.0 ; \quad \mathrm{MSE}=86.4 ; p<0.05)$, indicating less response errors with the same-amplitude display than with 
the different-amplitude display ( 7.7 vs. $12.4 \%$, respectively). Neither the main effect of the factor transition group nor any further interaction reached statistical significance. Separate ANOVAs for the pivot-repetition group and for the mapping-repetition group showed the main effect of transition condition to be significant only for the pivot-repetition group $(F(1,22)=14.0 ; \mathrm{MSE}=34.2 ; p<0.01)$ but not for the mapping-repetition group $(F<1)$. Neither the main effects of transition subgroup nor the interactions between transition condition and transition subgroup reached statistical significance.

\section{Discussion}

The goal of Experiment 2 was to investigate two-handed tool-use actions in a more natural setting. To do so, our strategy was twofold. First, we changed the response apparatus. In Experiment 2, participants had to move one of two sticks instead of pressing keys to control the lever displayed on the computer screen. Second, we decided to manipulate tool and mapping switches between-subjects, so that one group of participants always operated one and the same tool with both hands, whereas another group always acted with the same mapping. The results of Experiment 2 show that hand switch costs are larger for those participants who operated one and the same tool with different effector-specific mappings. Furthermore, participants of the other group, who always acted with the same mapping, responded faster overall, although they had to switch between different tools. This pattern of results is predicted by the hypothesis, that participants use a mapping-based representation instead of a mental model representing the physical tool at hand.

To control for amplitude differences of the imagined movement of the lever's handle, we used two different lever displays. In one of them, target points were placed in equal distance to the pivotal points, which lead to larger amplitudes of the imagined movement of the lever's handle with a compatible mapping than with an incompatible mapping. In the other one, target points were situated closer to the valid handle of the lever, thus ensuring that the amplitude of the imagined movement of the lever's handle is the same for compatible and incompatible target-to-movement mappings. Although the observations of Kunde (2003) suggest, that reaction times might be modulated by effect duration (i.e. the imagined movement of the lever's handle), the only observed significant effect was the main effect of display group in the analysis on error rates. Participants committed less response errors when they underwent the experiment with the target points situated closer to the valid handle of the lever. This result might be attributed to the fact, that participants could use the target points as an additional hand cue in this condition, which might have reduced errors in hand selection.
One might still wonder, however, if the implementation of the new response apparatus was sufficient to build up participants' impression of performing real tool-use actions. Two additional factors might have worked against such an impression. First, participants had to grip the response sticks with a precision grip between their index and thumb, whereas the lever displayed on the screen called for a power grip. These two handgrips differ in functional and phylogenetic aspects as well as in their associated cortical activity (Ehrsson et al., 2000; Napier, 1956). Therefore, it seems reasonable to assume that participants still had to translate the imagined bodily movement into the appropriate movement on the response apparatus. Second, if participants moved one stick (i.e. handle), they did not experience a movement of the other stick, because the two sticks were not connected. Moving one of the handles of a real lever, however, also leads to an associated passive movement of the other handle. This factor might work against the formation of a tool-based representation and therefore, the results obtained in Experiment 2 could still reflect cognitive processes of simulating tool use rather than cognitive processes of performing actions with a real tool at hand. Therefore, in Experiment 3 we implemented a real tool use setting, in which participants had to touch target locations in space with a movable lever.

\section{Experiment 3}

Experiment 3 was a replication of Experiment 2. As a change, participants were required to move the left or right handle of a real lever to touch one of two target locations (see Fig. 7). If the advantage of repeating the target-to-movement mapping over repeating the tool-mechanics observed so far, is due to participants simulating tool-use actions instead of

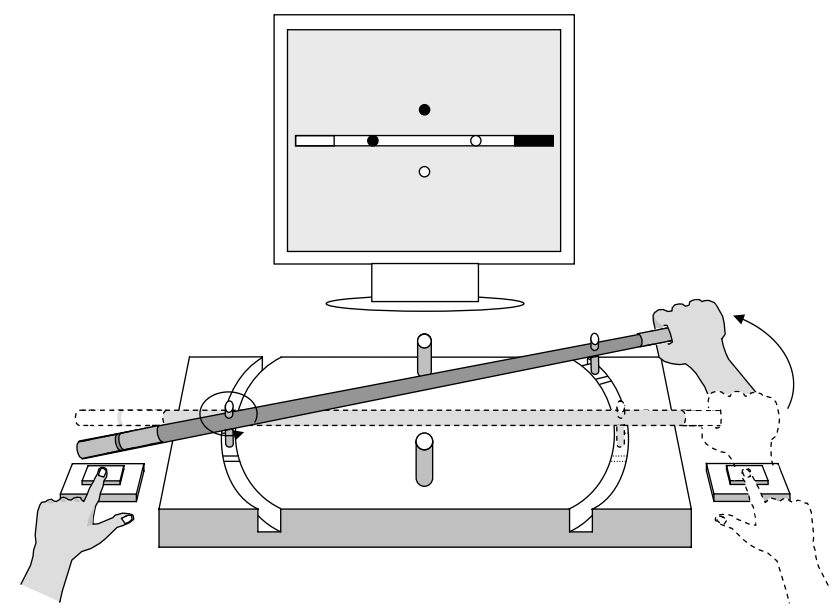

Fig. 7 Lever apparatus used in Experiment 3. The figure shows a compatible target-to-movement mapping (i.e. the handle has to be moved upwards to touch the upper target point) 
performing actions with a real tool at hand, then this advantage should disappear in Experiment 3. If, however, participants favour a mapping-based representation even when operating a real tool, then the observed advantage of a mapping repetition should also be apparent in Experiment 3.

\section{Method}

\section{Participants}

A total of 48 right-handed participants ( 29 female, 19 male; mean age $=24.0$ years), with normal or corrected-to-normal vision from the subject pool of the Max-Planck-Institute in Leipzig, participated in Experiment 3. They were randomly assigned to two groups of 24 participants each. Participants were naive to the purpose of the experiment and were paid for their participation.

\section{Apparatus and stimulus display}

The apparatus consisted of a metallic lever, which was mounted horizontally on a plastic plate $(45 \mathrm{~cm} \times 45 \mathrm{~cm})$. The lever (total length $75 \mathrm{~cm}$ with a diameter of $2 \mathrm{~cm}$ ) was movable around two possible pivotal points, placed in a distance of $37 \mathrm{~cm}$ to each other. Each pivotal point was in a distance of $19 \mathrm{~cm}$ from the end of the appropriate handle and could be locked and unlocked by a magnet attached beneath it. Unlocking one of the pivotal points made the lever movable around the locked pivotal point, whereas the lever was not movable at all, if both were locked. Moreover, there were two wired, elastic pins with a light diode on top of it serving as possible target points, which were mounted in a distance of $10 \mathrm{~cm}$ orthogonal to the lever. The target points could be touched with the lever by moving one of his handles towards or away from the body. Touching a target point caused turning on the diode on top of it. There were also light diodes on the top of the pivotal points which signalled, whether it was active at the moment. Furthermore, two home keys $(7 \mathrm{~cm} \times 6 \mathrm{~cm})$ were situated on the left and right side in front of the apparatus. The apparatus and the home keys were connected to a standard IBM-compatible computer via the parallel port. The pivotal points could be locked or unlocked by signals from the computer and the light diodes could be turned on and off. What is more, the computer registered which hand was used to control the lever (i.e. leaving the left or right home key) and whether the lever was in the resting position, left it in the direction toward or away from the body and touched the near or far target point.

\section{Design and procedure}

Design and procedure were the same as in Experiment 2 with the following exceptions. Participants were instructed to press down both home keys with the left and right index fingers, which triggered the beginning of the trial. After the presentation of the instruction display, participants were required to respond by moving the lever with the indicated hand while the other hand had to stay on the home key. Reaction time was defined as the time from the onset of the lever display to the leaving of the home key. Movement time was defined from leaving the home key to the arrival at the target point. Touching the target point triggered the $500 \mathrm{~ms}$ presentation of an associated effect display. The effect display showed the lever, moved around the active pivotal point, so that the participant was able to watch whether the instructed target point was touched by the lever. If the participant responded with the wrong hand, used both hands, or if the instructed target point was not touched, an error feedback was presented. After one of the target points had been touched, the lever was to be moved back to its resting position, all lights were turned off and both pivotal points were locked. The next trial started after participants pressed down both home keys.

The experiment consisted of a practice block of 16 trials, after which subjects went through six experimental blocks of 64 trials (plus one additional, later discarded starting trial). After each block, participants had the opportunity to take a short break. The whole experiment took approximately $40 \mathrm{~min}$.

\section{Results}

After excluding trials from the analysis in which participants responded in the preparation interval or responded with both hands (1.0\%), individual median reaction times for correct responses and error rates were calculated for hand repetitions and hand switches for each of the two transition groups (pivot-repetition group vs. mapping-repetition group). They are depicted in Fig. 8.

\section{$R T s$}

A repeated-measures ANOVA with the within-subject factor transition condition (hand-repetition/hand-switch) and the between-subject factor transition group (pivot-repetition/mapping-repetition) revealed a significant main effect of transition condition $(F(1,46)=16.4 ; \quad \mathrm{MSE}=375$; $p<0.001$ ), that was due to shorter reaction times on trials with a hand repetition. This main effect was qualified by a significant interaction of transition condition and transition group $(F(1,46)=13.8$; MSE $=375 ; p<0.01)$, demonstrating that hand switch costs differed between the two groups. Furthermore, the main effect of transition group $(F(1,46)=$ 4.2; $\mathrm{MSE}=13,147 ; p<0.05)$ reached statistical significance, indicating shorter reaction times in the mapping-repetition group. Simple interaction analysis for the pivot-repetition 
Fig. 8 Mean RT (in ms) on the left panel and mean error rates (in \%) on the right panel as a function of transition condition and transition group in Experiment 3. Error bars show standard errors

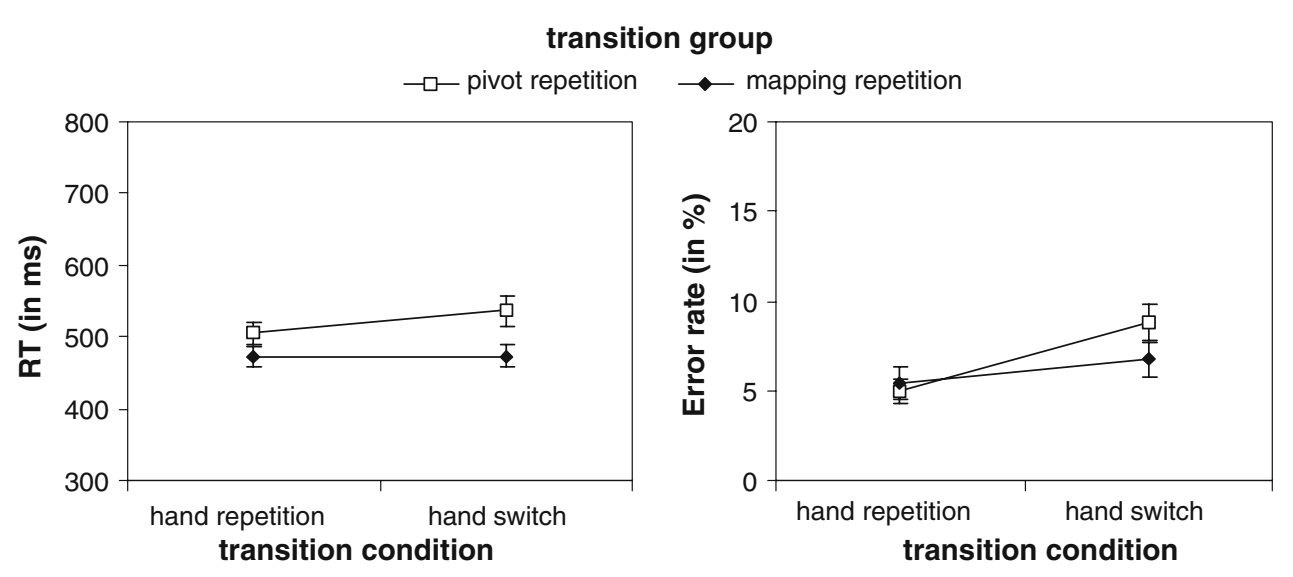

group and the mapping-repetition group with the factors transition condition and transition subgroup, revealed the main effect of transition condition to be significant only for the pivot-repetition group $(F(1,22)=20.3$; $\mathrm{MSE}=557$; $p<0.001)$ but not for the mapping-repetition group $(F<1)$. Neither the main effects of transition subgroup nor the interactions between transition condition and transition subgroup reached statistical significance.

To further test, whether the observed pattern of results was modulated by target transition (target-repetition/target switch) or hand (left hand/right hand), two separate ANOVAs were conducted. Entering target transition as an additional factor did not qualify the main effect of transition group and the interaction transition condition $\times$ transition group (all $F_{\mathrm{s}}<1$ ). However, the main effect of target-repetition $(493 \mathrm{~ms})$ versus target-switch $(502 \mathrm{~ms})(F(1,46)=$ 14.2; $\mathrm{MSE}=275 ; p<0.001)$ and the interaction transition condition $\times$ target transition reached statistical significance $(F(1,46)=42.2$; MSE $=236 ; p<0.001)$, indicating pronounced hand switch costs with a target repetition. Moreover, entering hand as an additional factor did not qualify any of the main effects or interactions.

\section{Error rates}

Analysis of error rates showed a transition condition main effect $(F(1,46)=12.7$; MSE $=12.4 ; p<0.01)$, that was due to less response errors on trials with a hand repetition. The interaction of transition condition $\times$ transition group missed the significance level $(F(1,46)=2.9 ; \operatorname{MSE}=12.4$; $p<0.10)$. The main effect of transition group was not significant either $(F<1)$.

Separate ANOVAs showed that neither the main effect of transition group nor the interaction transition condition $\times$ transition group was qualified by the factor target transition. The main effect of target transition (targetrepetition: $5.7 \%$ vs. target-switch: $7.2 \%)(F(1,46)=15.6$; MSE $=6.9 ; \quad p<0.001)$ and the interaction transition condition $\times$ target transition reached statistical significance
$(F(1,46)=19.3 ; \mathrm{MSE}=10.6 ; p<0.001)$. The interaction indicated pronounced hand switch costs with a target repetition. Entering hand (left hand/right hand) as an additional factor did not qualify any of the main effects or interactions.

\section{Discussion}

Experiment 3 replicated the results obtained in Experiment 2 in a real tool use setting. The results are clear-cut. Once again, the costs of switching hands were pronounced when participants operate one and the same tool with different effector-specific mappings. Furthermore, participants of the mapping-repetition group responded faster than participants of the pivot-repetition group. This pattern of results once again supports the hypothesis, that participants use a mapping-based representation instead of a mental model of the physical tool at hand.

There are studies in the literature on task-switching showing that switching one task dimension (e.g., the hand) can lead a switch benefit for another task dimension (e.g. the pivot), when the task is hierarchically structured, the second task dimension is lower in the hierarchy than the first, and both are switched concurrently (cf. Kleinsorge \& Heuer, 1999; Kleinsorge, Heuer \& Schmidtke, 2001). On the basis of these studies, it might be argued that our results can be interpreted in two different ways. The first interpretation is that the group with constant pivot shows worse performance, because in this group participants have to switch mappings when the hand is switched (this is the interpretation already given above). However, an alternative interpretation would be that this group shows worse performance because it is difficult to repeat the pivot, when the hand is switched. This interpretation would presuppose that there is a hierarchical representation of task dimensions, in which the pivot dimension is subordinate to the hand dimension (with the mapping not being represented).

Note, however, that this second interpretation is also incompatible with a tool-based representation in action planning with tools. In a tool-based representation, the tool 
(i.e. the pivot) should be highest in the hierarchy, and switch costs should be most pronounced in the group with changing pivot, regardless of whether the hand is switched or not. This pattern of results has not been found.

In our view, two reasons speak against an interpretation of the data pattern in terms of a cost for repeating the pivot together with switching the hand. The main reason is that for such a cost to occur, each task dimension must be varied along at least two possible values. If one task dimension can take only one value, there is no necessity to represent this dimension, as it does not change anyway. However, in both Experiments 2 and 3, for the group with high switch costs the pivot was constant throughout the block, and there was no dimensional organization of hand and pivot dimension (but a dimensional organization of hand and mapping dimension). Hence, there is no necessity to (strongly) represent the pivot and switch costs are likely to arise from the dimensional organization of hand and mapping. Second, we have found evidence for a strong representation of the mapping in a couple of prior studies on both unimanual and bimanual tool use, in which different experimental paradigms and measures (e.g. precuing, sequential effects) have been used (Massen \& Prinz, 2007a, b; Lepper et al., 2008). This converging evidence supports the interpretation that the high switch costs in the group with constant pivot are caused by switching the target-to-movement mapping.

Participants of Experiment 3 were considerably faster and more accurate (overall RT: $497 \mathrm{~ms}$; PE: 6.5\%) in comparison to participants of Experiment 2 (overall RT: $611 \mathrm{~ms}$; PE: 10.1\%). Although caution is warranted in comparing RTs and error rates across different experiments with different response devices, this effect could indicate that the implementation of a real tool use setting reduced the effort of translating the imagined bodily movement into the appropriate movement on the response apparatus. In a similar way, the smaller group differences in Experiment 3 as compared to Experiment 2 could indicate, that switching between different target-to-movement mappings of both hands is easier (although still more difficult than switching between different pivots) when the required mapping for each hand is immediately available from the response device.

Another interesting aspect of the results is that the target transition factor, that is whether there was a target repetition or a target switches from trial $n-1$ to trial $n$, neither modulated the group factor nor the interaction of transition condition $x$ transition group. From the viewpoint of the hypothesis of a tool-based representation, one might have predicted, that switching hands while operating one and the same tool should be especially easy when the target location is repeated. If participants automatically activate the corresponding movement of the non-responding hand when moving one of the lever handles to a target location, then repeating this target location while switching hands in the next trial should yield an advantage. However, in contrast to these considerations, the results obtained in Experiment 3 indicate reduced hand switch costs with a target switch regardless of group membership.

\section{General discussion}

In this study, we were interested in the mental processes and representations guiding two-handed tool use. More specifically, we asked whether it is easier to switch between different effector-specific target-to-movement mappings afforded by the same tool, or whether it is easier to switch between different tools, when the target-to-movement mapping is the same for both effectors. Across all three experiments, we found pronounced costs of switching hands, when a hand switch was associated with switching the target-to-movement mapping, even if the tool remains constant.

Regarding the hypotheses put forth in the introduction, the overall pattern of results strongly supports the mappingbased hypothesis. This hypothesis states that humans have quite abstract and tool-independent representations of hand-specific mappings in two-handed tool use. If both effectors share one common target-to-movement mapping, only one representation has to be specified. In contrast, operating one and the same tool with different effector-specific mappings requires the specification of two different representations. Therefore, a hand switch that is associated with a switch of the effector-specific target-to-movement mapping should be more demanding than a hand switch that is associated with a change of tool mechanics.

The validity of this conclusion is further confirmed by three facts. First, the results obtained are not limited to situations that might be regarded as rather artificial simulations of tool use. In fact, Experiment 3 clearly shows that the mapping-based hypothesis is supported even when participants acted in a more natural and ecologically valid setting and performed tool-use actions with a real lever. Second, as the results of Experiments 2 and 3 shows, there was no evidence for a higher order representation of a tool even if one group of participants always acted with the same tool. This observation is essential because manipulating tool and mapping switches between-subjects should be in principle enable participants to form a tool-based representation, which might take some time and experience with one and the same tool. The fact that this is not the case, once again shows that the mapping-based hypothesis holds even when a more natural way of using tools is applied. Finally, as the additional analysis of Experiment 3 suggests, the observed pattern of results is not constrained by whether the tool is operated with the 
dominant right or the non-dominant left hand. In studies about motor learning, a benefit of generalization to the contralateral hand is often reported, if the dominant hand initially experienced the task (Criscimagna-Hemminger, Donchin, Gazzaniga, \& Shadmehr, 2003; Thut et al., 1996). However, such transfer benefits are mostly observed for variables describing ongoing motor performance (e.g. trajectory accuracy or movement time) rather than movement selection. The fact that the present study neither finds effects of interest on movement time nor for whether the left or right hand is used, may be taken to demonstrate that the representations guiding two-handed tool use are relatively abstract and thus not enhanced with fine-grained information about movement execution.

The results of the present study are in accordance with the findings of Beisert et al. (2008). These authors showed that switching between different everyday tools is easier if the tools share a common target-to-movement mapping, which suggests that the cognitive representation of the target-to-movement mapping is relatively abstract and toolindependent. Our results extend this finding in several respects. First, in the study of Beisert et al. a tool-repetition was always associated with a mapping-repetition. In contrast, we manipulated tool and mapping switches independently which allowed us to disentangle tool and mapping repetition benefits. Second, we were able to extend the previous findings of the dominant role of the target-to-movement mapping in unimanual tool use into the domain of two-handed tool use.

It might be objected that we do not find evidence for tool-based representations in our experiments, because participants represent translational movements of two separate handles rather than a rotational movement of the lever. Especially in Experiments 1 and 2, responses of each hand followed a straight path and had no immediate effect on the part of the apparatus that was operated with the other hand. However, in Experiment 3, movements of one of the two handles were associated with a simultaneous movement of the other handle and the movements of the hands and handles were slightly curved. This should in principle promote a rotational representation of the lever. The fact that costs for switching hands in Experiment 3 were reduced as compared to Experiment 2 can be interpreted as evidence that participants did indeed represent the lever as a two-handed, rotating tool in Experiment 3. Furthermore, especially in the condition with the incompatible target-to-movement mapping participants were forced to pay attention to the rotational movement of the lever's distal part and its reaching of the target position, because otherwise they would not have known when to stop the movement. Hence we think that the apparatus used in Experiment 3 promotes a representation of the lever as a two-handed, rotating tool. However, of course these considerations do not rule out the possibility that there are other environmental or experimental conditions that would lead participants to adopt a tool-based representation.

The latter point might also be related to a different line of research that is concerned with the learning of object dynamics during grasping (Edin, Westling, \& Johansson, 1992; Gordon, Forssberg, \& Iwasaki, 1994; Johansson \& Westling, 1984; Salimi, Hollender, Frazier, \& Gordon, 2000). Measuring grip force modulation while participants performed successive lifts with different effectors, some of these studies were able to show a transfer of information about the object's texture (Johansson \& Westling, 1984) and weight (Gordon, Forssberg, \& Iwasaki, 1994). However, other studies (Edin, Westling, \& Johansson, 1992; Salimi, Hollender, Frazier, \& Gordon, 2000) failed to show transfer effects between effectors. For instance, Salimi et al. (2000) measured the anticipatory control of fingertip forces while participants were grasping an object with a shifted weight distribution. The authors found that within three to five lifts, participants were able to adjust their load force before lift-off so that the object was lifted without rolling sideways. However, despite participants' explicit knowledge of a hand switch, the already achieved information about the weight distribution was not transferred to the other hand, resulting in a subsequent large roll of the object. The latter result suggests that the representation of the object's weight distribution is specific to the effectors used to form it. To sum up, it still remains unclear, whether an object-based internal representation or an effector-based representation is used for anticipatory control of fingertip forces. However, our results seem to indicate that in two-handed tool use the transfer of information between effectors might not be boosted by the coherence of an object (or tool) but rather by the coherence of the effector-specific mappings required to achieve a desired goal.

To summarize, this paper has presented evidence for the notion that two-handed tool-use actions are not automatically represented with respect to the underlying mechanics of the tool, but rather in terms of their hand-specific targetto-movement mappings. This finding extends existing evidence for rather abstract tool representations in unimanual tool use.

Acknowledgments We would like to thank Henrik Grunert for constructing the lever device and Yvonne Semek and Sascha Weis for their help with data acquisition. We also thank Miriam Beisert for providing presentation routines for trial randomization. This work has been financially supported by DFG-grant MA 2584/2-1 to Cristina Massen.

Open Access This article is distributed under the terms of the Creative Commons Attribution Noncommercial License which permits any noncommercial use, distribution, and reproduction in any medium, provided the original author(s) and source are credited. 


\section{References}

Beisert, M., Massen, C., \& Prinz, W. (2008). Embodied rules in tool use: A tool-switching study. (submitted).

Criscimagna-Hemminger, S. E., Donchin, O., Gazzaniga, M. S., \& Shadmehr, R. (2003). Learned dynamics of reaching movements generalize from dominant to nondominant arm. Journal of Neurophysiology, 89, 168-176.

Diedrichsen, J., Ivry, R. B., Hazeltine, E., Kennerly, S., \& Cohen, A. (2003). Bimanual interference associated with the selection of target locations. Journal of Experimental Psychology: Human Perception \& Performance, 29, 64-77.

Edin, B. B., Westling, G., \& Johansson, R. S. (1992). Independent control of human finger-tip forces at individual digits during precision lifting. Journal of Physiology, 450, 547-564.

Ehrsson, H. H., Fagergren, A., Jonsson, T., Westling, G., Johansson, R. S., \& Forssberg, H. (2000). Cortical activity in precision-versus power-grip tasks: An fMRI study. Journal of Neurophysiology, $83,528-536$

Gordon, A. M., Forssberg, H., \& Iwasaki, N. (1994). Formation and lateralization of internal representations underlying motor commands during precision grip. Neuropsychologia, 32, 555-568.

Guiard, Y. (1983). The lateral coding of rotations: A study of the Simon effect with wheel-rotation responses. Journal of Motor Behavior, 15, 331-342.

Heuer, H. (1993). Structural constraints on bimanual movements. Psychological Research, 55, 83-98.

Heuer, H., \& Klein, W. (2006). The influence of movement cues on intermanual interactions. Psychological Research, 70, 229-244.

Johansson, R. S., \& Westling, G. (1984). Roles of glabrous skin receptors and sensorimotor memory in automatic control of precision grip when lifting rougher or more slippery objects. Experimental Brain Research, 56, 550-564.

Johnson-Frey, S. H. (2003). What's so special about human tool use? Neuron, 39, 201-204.

Kleinsorge, T., \& Heuer, H. (1999). Hierarchical switching in a multidimensional task space. Psychological Research, 62, 300-312.

Kleinsorge, T., Heuer, H., \& Schmidtke, V. (2001). Task-set reconfiguration with binary and three-valued task dimensions. Psychological Research, 65, 192-201.

Kunde, W. (2003). Temporal response-effect compatibility. Psychological Research, 67, 153-159.

Kunde, W., \& Weigelt, M. (2005). Goal congruency in bimanual object manipulation. Journal of Experimental Psychology: Human Perception \& Performance, 31, 145-156.

Kunde, W., Müsseler, J., \& Heuer, H. (2007). Spatial compatibility effects with tool use. Human Factors, 49, 661-670.
Lepper, M., Massen, C., \& Prinz, W. (2008). What to do and how to do it: Sequence learning of actions effects and transformation rules. Acta Psychologica, 128, 139-152.

Loftus, G. R., \& Masson, M. E. J. (1994). Using confidence intervals in within-subject designs. Psychonomic Bulletin \& Review, 1, 476-490.

Massen, C., \& Prinz, W. (2007a). Programming tool-use actions. Journal of Experimental Psychology: Human Perception \& Performance, 33, 692-704.

Massen, C., \& Prinz, W. (2007b). Activation of action rules in action observation. Journal of Experimental Psychology: Learning, Memory \& Cognition, 33, 1118-1130.

Müsseler, J., Kunde, W., Gausepohl, D., \& Heuer, H. (2008). Does a tool eliminate spatial compatibility effects? European Journal of Cognitive Psychology, 20, 211-231.

Napier, J. R. (1956). The prehensile movements of the human hand. Journal of Bone \& Joint Surgery, 38B, 902-913.

Proctor, R. W., Wang, D.-Y. D., \& Pick, D. F. (2004). Stimulus-response compatibility with wheel-rotation responses: Will an incompatible response coding be used when a compatible coding is possible? Psychonomic Bulletin \& Review, 11, 841-847.

Rosenbaum, D. A. (1980). Human movement initiation: Specification of arm, direction, and extent. Journal of Experimental Psychology: General, 109, 444-474.

Rosenbaum, D. A. (1983). The movement precuing technique: Assumptions, applications, and extensions. In R. A. Magill (Ed.) Memory and control of action (pp. 231-274). Amsterdam: NorthHolland.

Salimi, I., Hollender, I., Frazier, W., \& Gordon, A. M. (2000). Specificity of internal representations underlying grasping. Journal of Neurophysiology, 84, 2390-2397.

Spijkers, W., Heuer, H., Kleinsorge, T., \& van der Loo, H. (1997). Preparation of bimanual movements with same and different amplitudes: Specification interference as revealed by reaction time. Acta Psychologica, 96, 207-227.

Thut, G., Cook, N. D., Regard, M., Leenders, K. L., Halsband, U., \& Landis, T. (1996). Intermanual transfer of proximal and distal motor engrams in humans. Experimental Brain Research, 108, 321327.

Wang, D.-Y. D., Proctor, R. W., \& Pick, D. F. (2003). The Simon effect with wheel-rotation responses. Journal of Motor Behavior, 35, 261-273.

Wang, D.-Y. D., Proctor, R. W., \& Pick, D. F. (2007). Coding controlled and triggered cursor movements as action effects: Influences on the auditory Simon effect for wheel-rotation responses. Journal of Experimental Psychology: Human Perception and Performance, 33, 657-669. 